\title{
考虑区间不确定性的双摆吊车运动轨迹规划
}

\author{
彭海军 $^{1}$ 施博洋 ${ }^{1}$ 王昕炜 $^{1}$ 谢小辉 $^{2}$ 孙立宁 $^{2}$
}

(1. 大连理工大学工业装备结构分析国家重点实验室 大连 116024;

2. 苏州大学机电工程学院 苏州 215006)

\begin{abstract}
摘要: 不确定因素会使吊车运动偏离预定轨迹, 导致严重安全事故。为在轨迹规划时研究不确定性对吊车运动的影响, 提出 一种基于区间模型的不确定性研究方法。该方法首先采用区间模型描述不确定性, 形成区间形式的不确定性轨迹规划问题, 使用保辛伪谱方法进行采样, 并提出 Chebyshev 代理模型法(CSMM)近似原轨迹规划问题, 通过扫描代理模型求得不确定性 区间结果。在数值算例中, 利用该方法求解吊车的运动轨迹区间, 并分析不确定度对运动轨迹的影响。对比其他方法, 所提 出方法具有计算成本低、结果精度令人满意的特点。所提出的方法可快速准确求出不确定性下吊车运动轨迹区间, 直观表达 不确定性对吊车运动轨迹的影响, 为吊车系统的稳定性与鲁棒性提供参考。
\end{abstract}

关键词: 吊车; 区间不确定性; 最优控制; 轨迹规划; 代理模型

中图分类号: TP242

\section{Trajectory Planning of Double Pendulum Crane Considering Interval Uncertainty}

\author{
PENG Haijun $^{1}$ SHI Boyang ${ }^{1} \quad$ WANG Xinwei $^{1} \quad$ XIE Xiaohui $^{2} \quad$ SUN Lining ${ }^{2}$ \\ (1. State Key Laboratory of Structural Analysis for Industrial Equipment, \\ Dalian University of Technology, Dalian 116024; \\ 2. School of Mechanical and Electric Engineering, Soochow University, Suzhou 215006)
}

\begin{abstract}
Uncertain factors make the crane deviate from the predetermined trajectory, which leads to serious security accidents. In order to investigate the impact of uncertainty on the crane movement in the stage of trajectory planning, an uncertain method based on interval model is proposed. Firstly, uncertainty is described by interval model and an uncertain trajectory planning problem in the forms of interval is established.Then,a symplectic pseudospectral methodis used to sample and Chebyshev surrogate model method (CSMM) is proposed to approximate the original trajectory planning. The uncertain interval results are obtained by scanning surrogate model. In numerical examples, the intervals of crane movement trajectory are obtained by the proposed method and the impact of uncertain level on crane movement trajectory is analyzed.Compared with other methods, the proposed method has characteristic of low computational cost and satisfactory accuracy.The intervals of crane movement trajectory under uncertainty can be obtained via the proposed method, the impact of uncertainty on crane movement is intuitively expressed,which offer the reference for stability and robustness of the crane system.
\end{abstract}

Key words: crane; interval uncertainty; optimal control; trajectory planning; surrogate model

\section{0 前言}

作为典型的工业机器人, 吊车系统在诸如港口、 工厂、建筑工地等领域均有广泛的应用。根据动力 学特性以及坐标系统的差异, 吊车系统可以分为三 类: 桥式吊车、塔式吊车以及吊杆吊车 ${ }^{[1]}$ 。由于吊

* 国家自然科学基金资助项目(11472069, 91648204, 61473200)。20170515 收到初稿, 20180709 收到修改稿
车系统的控制输入维数小于待控制自由度的维数, 所以吊车系统是典型的欠驱动系统 ${ }^{[2]}$, 相较之全驱 动系统, 欠驱动系统更难以控制 ${ }^{[3]}$, 实际吊车系统 往往由经验丰富的工人操作, 若操作失误, 可能引 起碰撞、负载滑落等严重的安全事故。因此, 对吊 车系统的自动控制研究一直是研究人员关注的热 点, 并提出了许多动力学模型和相应的控制方法 ${ }^{[4]}$ 。

工业机器人 ${ }^{[5]}$ 的轨迹规划在诸多领域都得到广 泛应用, 吊车系统也不例外。规划轨迹时, 常利用 
最优控制的思想, 优化性能指标而又兼顾约束。研 究时, 一方面为提高生产效率, 有学者将吊车简化 为单摆模型 ${ }^{[6-7]}$, 进行了时间最优的运动轨迹规划, 但负载几何外形较大时, 这一简化是不符合实际的, 一些学者 ${ }^{[8-9]}$ 则使用双摆模型进行了改进; 另一方 面, 为降低生产成本, WU 等 ${ }^{[7,10]}$ 考虑了以优化能耗 为目标的运动规划问题。总之, 在开环设计阶段利 用最优控制的方法就可完成吊车的轨迹规划, 使其 按照预定轨迹运行, 一方面优化性能指标, 另一方 面也能减轻在线控制的负担。然而, 吊车运行时, 由于测量、生产、简化以及外部扰动等因素, 实际 吊车系统不可避免地存在不确定性, 不确定性会干 扰吊车运动, 使其偏离预定轨迹。为处理此问题, 一些研究人员尝试用在线反馈控制来应对不确定 性, FANG 等 ${ }^{[3]}$ 利用自适应控制结合李雅普诺夫方 法设计了控制方案, 而刘殿通等 ${ }^{[11]}$ 则结合模糊理论 使用滑模控制的方法处理不确定性的影响, TUAN 等 ${ }^{[12]}{ }^{+}$至还考虑了三维运动情况。综上可知, 当前 研究的吊车轨迹规划均针对确定性模型, 鲜有考虑 不确定性的轨迹规划。若仅依靠在线反馈的方法提 高吊车定位精度并补偿不确定性的干扰, 则增加了 在线实时控制的负担, 因此考虑不确定性的吊车轨 迹规划是非常必要的。

就不确定性而言, 目前已存在许多研究方法, 其中基于区间理论 ${ }^{[13]}$ 的方法由于不需要原问题的统 计信息且不依赖原问题的具体求解方法, 具有很强 的通用性, 可用于处理常微分方程(ODEs) 问题 ${ }^{[14-15]}$ 、 微分代数方程(DAEs) 问题 ${ }^{[16]}$ 、以及参数优化问题 ${ }^{[17]}$, 且使用区间理论处理不确定性问题时, 往往与代理 模型方法配合使用, 可以最大限度地减少计算时间。

结合以上研究现状, 考虑到不确定性对吊车轨 迹规划所带来的困难, 本文提出了 Chebyshev 代理 模型法(CSMM)。在吊车离线规划轨迹时就考虑不 确定性对其运动的影响, 预示吊车在实际运动时的 轨迹变化, 分析不确定参数对系统的影响, 为鲁棒 性与稳定性研究提供参考。具体而言, 首先使用基 于最优控制的保辛伪谱方法 ${ }^{[18]}$ 求解吊车的轨迹规 划, 并采用逼近效果良好的 Chebyshev 级数作为代 理模型逼近原轨迹规划问题, 用简单的多项式代替 需要多次迭代才能求解的最优控制问题, 进而将吊 车轨迹规划的参数作为输入参数, 而将所有轨迹信 息作为响应, 考察不确定性时, 使用输入参数的分 布区间模拟外界不确定性, 最终通过遍历代理模型, 可求出不确定参数的响应区间, 并分析不同参数对 吊车系统的影响程度以及同一参数在不确定性变化 时对吊车系统的影响。数值结果表明本文所提出的
方法具有响应区间准确、计算效率高的优点。

\section{1 吊车模型及其轨迹规划}

\section{1 双摆吊车的动力学模型}

对于图 1a 所示的桥式吊车, 可以合理简化为如 图 1b 所示的双摆模型, 其中吊车质量为 $M$, 吊钩 质量为 $m_{1}$, 重物质量为 $m_{2}$, 吊车位移为 $x$, 吊钩摆 角为 $\theta_{1}$, 重物摆角为 $\theta_{2}$, 吊车质心至吊钩质心的距 离为 $l_{1}$, 吊钩质心至重物质心的距离为 $l_{2}$, 施加于 小车上的控制力为 $u$ 。系统的控制方程为

$$
D(q) \ddot{q}+C(q, \dot{q})+G(q)=U
$$

式中, $\boldsymbol{q}$ 代表所选的广义坐标有 $\boldsymbol{q}=\left(x, \theta_{1}, \theta_{2}\right)^{\mathrm{T}}$ 。矩 阵 $\boldsymbol{D}(\boldsymbol{q}), \boldsymbol{C}(\boldsymbol{q}, \dot{\boldsymbol{q}}), \boldsymbol{G}(\boldsymbol{q})$ 分别为

$$
\begin{gathered}
\boldsymbol{D}(\boldsymbol{q})=\left(\begin{array}{lll}
d_{11} & d_{12} & d_{13} \\
d_{21} & d_{22} & d_{23} \\
d_{31} & d_{32} & d_{33}
\end{array}\right) \\
\left\{\begin{array}{l}
d_{11}=M+m_{1}+m_{2} \\
d_{12}=d_{21}=\left(m_{1}+m_{2}\right) l_{1} \cos \theta_{1} \\
d_{13}=d_{31}=m_{2} l_{2} \cos \theta_{2} \\
d_{22}=\left(m_{1}+m_{2}\right) l_{1}^{2} \\
d_{23}=d_{32}=m_{2} l_{1} l_{2} \cos \left(\theta_{1}-\theta_{2}\right) \\
d_{33}=m_{2} l_{2}^{2}
\end{array}\right.
\end{gathered}
$$

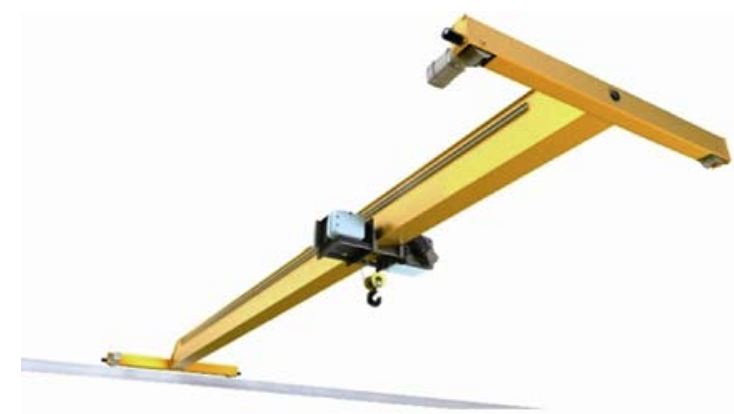

(a) 桥式吊车示意图

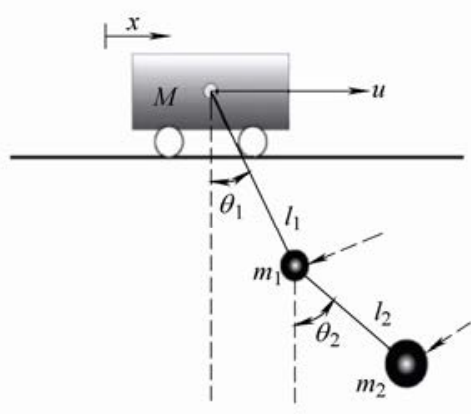

(b) 双摆模型

图 1 桥式吊车及其简化模型

$$
\boldsymbol{C}(\boldsymbol{q}, \dot{\boldsymbol{q}})=\left(\begin{array}{lll}
c_{11} & c_{12} & c_{13} \\
c_{21} & c_{22} & c_{23} \\
c_{31} & c_{32} & c_{33}
\end{array}\right)
$$




$$
\begin{aligned}
& \left\{\begin{array}{l}
c_{11}=c_{21}=c_{22}=c_{31}=c_{33}=0 \\
c_{12}=-\left(m_{1}+m_{2}\right) l_{1} \dot{\theta}_{1} \sin \theta_{1} \\
c_{13}=-m_{2} l_{2} \dot{\theta}_{2} \sin \theta_{2} \\
c_{23}=m_{2} l_{1} l_{2} \dot{\theta}_{1} \sin \left(\theta_{1}-\theta_{2}\right) \\
c_{32}=-m_{2} l_{1} l_{2} \dot{\theta}_{1} \sin \left(\theta_{1}-\theta_{2}\right)
\end{array}\right. \\
& \boldsymbol{G}(\boldsymbol{q})=\left(\begin{array}{lll}
0 & \left(m_{1}+m_{2}\right) g l_{1} \sin \theta_{1} & m_{2} g l_{2} \sin \theta_{2}
\end{array}\right)^{\mathrm{T}} \\
& \boldsymbol{U}=\left(\begin{array}{lll}
u & 0 & 0
\end{array}\right)^{\mathrm{T}}
\end{aligned}
$$

若在状态空间进行描述, 引入状态矢量 $\boldsymbol{x}=\left(\begin{array}{llllll}x & \dot{x} & \theta_{1} & \dot{\theta}_{1} & \theta_{2} & \dot{\theta}_{2}\end{array}\right)^{\mathrm{T}}$, 则原系统方程式(1)可 以化为

$$
\dot{\boldsymbol{x}}=\boldsymbol{f}(\boldsymbol{x}, u, t)
$$

式(1)或式(8)为吊车的动力学方程。

\section{2 吊车的轨迹规划}

实际工程中, 吊车系统出于安全性以及工作效 率的考虑, 还存在相应的物理约束。在本文中, 将 考虑以下三方面的约束。

(1) 吊车系统起始与终止时, 为保证起吊与卸 下重物作业安全, 要求起始时刻 $t_{0}$ 时, 吊车位移和 速度为 0 , 吊钩摆角和重物摆角为 0 , 且其角速度也 均为 0 。在吊车到达目标位置对应时刻 $t_{f}$, 除吊车 位移为给定值 $x_{f}$ 外, 其余各状态量均为 0 。用状态 矢量表示的两端边界条件为

$$
\begin{gathered}
x\left(t_{0}\right)=\boldsymbol{x}_{0}=\left(\begin{array}{llllll}
0 & 0 & 0 & 0 & 0 & 0
\end{array}\right)^{\mathrm{T}} \\
\boldsymbol{x}\left(t_{f}\right)=\boldsymbol{x}_{f}=\left(\begin{array}{llllll}
x_{f} & 0 & 0 & 0 & 0 & 0
\end{array}\right)^{\mathrm{T}}
\end{gathered}
$$

（2）考虑吊车运输过程中的安全性以及吊车电 机的实际特性, 吊车速度与输入控制力应保持在适 当范围内, 即

$$
|\dot{x}| \leqslant v_{\text {max }} \quad|u| \leqslant u_{\text {max }}
$$

（3）吊车运行过程中重物与吊钩的摆动可能会 引起不必要的碰撞, 同时摆动角度过大, 货物也有 脱落的危险。因此两级摆角及其角速度均应该保持 在给定范围内，有

$$
\begin{array}{ll}
\left|\theta_{1}\right| \leqslant \theta_{1 \max } & \left|\theta_{2}\right| \leqslant \theta_{2 \max } \\
\left|\dot{\theta}_{1}\right| \leqslant \omega_{1 \max } & \left|\dot{\theta}_{2}\right| \leqslant \omega_{2 \max }
\end{array}
$$

在本文中, 考虑最优化吊车能耗的策略, 吊车的能 耗可用以下成本函数 $J(u)$ 表征

$$
J(u)=\int_{0}^{t_{f}} u^{2} \mathrm{~d} t
$$

整个轨迹规划问题可以描述为: 寻找控制力函数 $u(t)$, 使吊车在满足前述约束条件的前提下, 成本 函数最小。寻找合适的 $u(t)$ 构成以下优化问题

\section{find $u$}

$\min J(u)$

s.t. $\dot{\boldsymbol{x}}=\boldsymbol{f}(\boldsymbol{x}, u, t)$

$$
\begin{aligned}
& \boldsymbol{x}\left(t_{0}\right)=\boldsymbol{0}, \boldsymbol{x}\left(t_{f}\right)=\left(\begin{array}{llllll}
x_{f} & 0 & 0 & 0 & 0 & 0
\end{array}\right)^{\mathrm{T}} \\
& |\dot{x}| \leqslant v_{\max } \quad|u| \leqslant u_{\max } \quad\left|\theta_{1}\right| \leqslant \theta_{1 \max } \quad\left|\theta_{2}\right| \leqslant \theta_{2 \max } \\
& \left|\dot{\theta}_{1}\right| \leqslant \omega_{1 \max } \quad\left|\dot{\theta}_{2}\right| \leqslant \omega_{2 \max }
\end{aligned}
$$

这是一个典型的最优控制问题，吊车系统参数给定 后, 求解该问题, 可以得到最佳输入控制力 $u(t)$ 保 证吊车运行时耗能最少, 进而吊车的轨迹规划也随 之完成。事实上，问题式(15)也可写为更一般的最 优控制问题的形式

$$
\begin{aligned}
& \text { find } \boldsymbol{u} \\
& \min J(\boldsymbol{u})=\int_{t_{0}}^{t_{f}} \boldsymbol{\Phi}(\boldsymbol{x}, \boldsymbol{u}, t) \mathrm{d} t \\
& \text { s.t. } \dot{\boldsymbol{x}}=\boldsymbol{f}(\boldsymbol{x}, \boldsymbol{u}, t) \\
& \boldsymbol{x}\left(t_{0}\right)=\boldsymbol{x}_{0}, \boldsymbol{x}\left(t_{f}\right)=\boldsymbol{x}_{f} \\
& h(x, u, t) \leqslant 0
\end{aligned}
$$

式中, $\dot{\boldsymbol{x}}=\boldsymbol{f}(\boldsymbol{x}, \boldsymbol{u}, t)$ 为系统的状态方程, $\boldsymbol{h}$ 表示 $q$ 维 不等式约束, $\boldsymbol{x}$ 和 $\boldsymbol{u}$ 分别表示系统的状态矢量和控 制输入矢量, $J(\boldsymbol{u})$ 为对应的性能指标。问题式(15)、 (16)中, 参数事先均已确定, 称之为确定性最优控 制问题。

对问题式(15)或(16)构成的确定性最优控制问 题, 本文采用保辛伪谱方法求解 ${ }^{[18]}$, 由于本文使用 最优控制的思想完成吊车运动轨迹规划, 为统一起 见后文中将吊车轨迹规划问题统一称为最优控制 问题。

\section{2 不确定性最优控制问题的区间分析}

问题式(15)尚未考虑不确定性, 本节将首先用 区间参数模拟吊车实际运行中的不确定性并建立相 应的不确定性最优控制问题, 再利用代理模型进行 求解。

\section{1 不确定性最优控制问题}

对于实际工程中的不确定性因素, 可以利用不 确定参数对其进行模拟。对于 $r$ 个不确定参数, 可 用列矢量 $\boldsymbol{\xi}=\left(\xi_{1}, \xi_{2}, \cdots, \xi_{r}\right)^{\mathrm{T}}$ 表示, 参数的变化可用包 络其变化范围的区间表达[16], 不确定参数的包络 区间矢量可记为 $\xi^{\mathrm{I}}=[\xi]=[\underline{\xi}, \bar{\xi}], \bar{\xi}$ 表示区间上限, $\overline{\boldsymbol{\xi}}=\left[\bar{\xi}_{1}, \cdots, \bar{\xi}_{r}\right]^{\mathrm{T}}, \quad \boldsymbol{\xi}$ 表示区间下限, $\boldsymbol{\xi}=\left(\xi_{1}, \cdots, \xi_{r}\right)^{\mathrm{T}}$, 有 $\xi \in \xi^{\mathrm{I}}$ 。该不确定参数区间矢量也可记为: $\xi^{1}=\left\langle\xi^{0}, \xi^{\mathrm{w}}\right\rangle$, 其中 $\xi^{0}$ 为 “中点值” 矢量, $\xi^{\mathrm{w}}$ 为 “半 径” 矢量。有 


$$
\xi^{0}=\frac{\underline{\xi}+\bar{\xi}}{2} \quad \xi^{\mathrm{w}}=\frac{\bar{\xi}-\underline{\xi}}{2}
$$

衡量不确定度时, 可利用不确定度的概念, 第 $i$ 个 参数的不确定度 $\gamma_{i}$ 定义为

$$
\gamma_{i}=\frac{\xi_{i}^{w}}{\left|\xi_{i}^{0}\right|}
$$

由于矢量区间 $\xi^{\mathrm{I}}$ 包含了不确定参数所有可能 的结果，用该区间就可代表不确定参数。对于问题 (16), 其中若含不确定参数, 吊车运行将受其影响, 即 $\forall \boldsymbol{\xi} \in \boldsymbol{\xi}^{\mathrm{I}}$, 均有 $J=J(\boldsymbol{u}, \boldsymbol{\xi}), \boldsymbol{u}=\boldsymbol{u}(\boldsymbol{\xi}, t), \boldsymbol{x}=\boldsymbol{x}(\boldsymbol{\xi}, t)$ 。 若不确定参数用区间表示, 且 $\forall \xi \in \xi^{\mathrm{I}}$, 问题(16)均 能求解, 则求解结果也构成区间, 有

$$
\begin{gathered}
\boldsymbol{u}=\left\{\begin{array}{cc}
\boldsymbol{u}(\boldsymbol{\xi}, t): \min _{\boldsymbol{u}} J(\boldsymbol{\xi}, \boldsymbol{u}) & \boldsymbol{\xi} \in \boldsymbol{\xi}^{\mathrm{I}} \\
\text { s.t. } \dot{\boldsymbol{x}}=\boldsymbol{f}(\boldsymbol{x}, \boldsymbol{u}, \boldsymbol{\xi}, t) & \\
\boldsymbol{x}\left(t_{0}\right)=\boldsymbol{x}_{0}, \boldsymbol{x}\left(t_{f}\right)=\boldsymbol{x}_{f} \\
\boldsymbol{h}(\boldsymbol{x}, \boldsymbol{u}, \boldsymbol{\xi}, t) \leqslant \boldsymbol{0}
\end{array}\right\} \\
\boldsymbol{x}=\left\{\begin{array}{cc}
\boldsymbol{x}(\boldsymbol{\xi}, t): \min _{\boldsymbol{u}} J(\boldsymbol{\xi}, \boldsymbol{u}) \\
\text { s.t. } \dot{\boldsymbol{x}}=\boldsymbol{f}(\boldsymbol{x}, \boldsymbol{u}, \boldsymbol{\xi}, t) \\
\boldsymbol{x}\left(t_{0}\right)=\boldsymbol{x}_{0}, \boldsymbol{x}\left(t_{f}\right)=\boldsymbol{x}_{f} \\
\boldsymbol{h}(\boldsymbol{x}, \boldsymbol{u}, \boldsymbol{\xi}, t) \leqslant \boldsymbol{0}
\end{array}\right\}
\end{gathered}
$$

式中, $\boldsymbol{u}$ 为输入控制区间矢量, $\boldsymbol{x}$ 为状态量区间 矢量。

由于整个优化过程在给定不确定参数区间后可 得到控制输入及状态量的分布区间, 可以利用区间 数学对其进行描述。根据区间理论, 若记 $\mathbf{R}^{n}$ 为 $n$ 维 实数集合而 $\mathbf{I R}^{n}$ 为包含所有实数 $\mathbf{R}^{n}$ 的区间集合, 当 $\boldsymbol{x} \in \mathbf{R}^{n},[\boldsymbol{x}] \in \mathbf{I R}^{n}$, 又有 $\boldsymbol{f}\left(\mathbf{R}^{n} \rightarrow \mathbf{R}^{m}\right)$, 则可以得 到 $\boldsymbol{f}$ 对应的区间扩张函数 $[\boldsymbol{f}]\left(\mathbf{I R}^{n} \rightarrow \mathbf{I R}^{\mathrm{m}}\right)$, 有

$$
[\boldsymbol{f}]=[\boldsymbol{f}]([\boldsymbol{x}])
$$

该函数中, 自变量 $[\boldsymbol{x}]$ 和函数 $[\boldsymbol{f}]$ 均为区间。利用区 间表达, 式(16)对应的最优控制问题变为

$$
\begin{aligned}
& \text { find }[\boldsymbol{u}] \\
& \min [J]([\xi],[\boldsymbol{u}]) \\
& \text { s.t. } \quad \dot{\boldsymbol{x}}=\boldsymbol{f}(\boldsymbol{x}, \boldsymbol{u}, \boldsymbol{\xi}, t) \\
& \boldsymbol{x}\left(t_{0}\right)=\boldsymbol{x}_{0}, \boldsymbol{x}\left(t_{f}\right)=\boldsymbol{x}_{f} \\
& \boldsymbol{h}(\boldsymbol{x}, \boldsymbol{u}, \boldsymbol{\xi}, t) \leqslant 0
\end{aligned}
$$

对问题式(22)需要做两点说明。

(1) $\min [J]$ 表示参数变化时, 所有 $J$ 的极小值 所构成的区间而非 $J$ 区间中的最小值。有: $\min [J]=[\min J]=[\min J, \overline{\min J}] \neq \min ([J])$, 其中 区间边界 $\underline{\min J}, \overline{\min J}$ 分别满足式(23)和(24)

$$
\underline{\min J}=\min _{\xi}\left\{\begin{array}{c}
J(\boldsymbol{\xi}, \boldsymbol{u}): \min _{\boldsymbol{u}} J(\boldsymbol{\xi}, \boldsymbol{u}) \\
\text { s.t. } \dot{\boldsymbol{x}}=\boldsymbol{f}(\boldsymbol{x}, \boldsymbol{u}, \boldsymbol{\xi}, t) \\
\boldsymbol{x}\left(t_{0}\right)=\boldsymbol{x}_{0}, \boldsymbol{x}\left(t_{f}\right)=\boldsymbol{x}_{f} \\
\boldsymbol{h}(\boldsymbol{x}, \boldsymbol{u}, \boldsymbol{\xi}, t) \leqslant \mathbf{0}
\end{array}\right\}
$$

$$
\overline{\min J}=\max _{\xi}\left\{\begin{array}{c}
J(\boldsymbol{\xi}, \boldsymbol{u}): \min _{\boldsymbol{u}} J(\boldsymbol{\xi}, \boldsymbol{u}) \\
\text { s.t. } \dot{\boldsymbol{x}}=f(\boldsymbol{x}, \boldsymbol{u}, \boldsymbol{\xi}, t) \\
\boldsymbol{x}\left(t_{0}\right)=\boldsymbol{x}_{0}, \boldsymbol{x}\left(t_{f}\right)=\boldsymbol{x}_{f} \\
\boldsymbol{h}(\boldsymbol{x}, \boldsymbol{u}, \boldsymbol{\xi}, t) \leqslant \boldsymbol{0}
\end{array}\right\}
$$

(2) 约束条件中 $\dot{\boldsymbol{x}}=\boldsymbol{f}(\boldsymbol{x}, \boldsymbol{u}, \boldsymbol{\xi}, t), \boldsymbol{h}(\boldsymbol{x}, \boldsymbol{u}, \boldsymbol{\xi}, t) \leqslant 0$ 表示 $\forall \xi \in[\xi]$, 公式均成立, 两个公式中的函数为 点函数并非区间扩张函数。

由上述分析可见，与确定性最优控制问题不同， 不确定性问题的解为区间而不再是一个点解, 后文 将介绍如何求得解区间。

\subsection{Chebyshev 代理模型}

求解问题式(22)前，注意到尽管问题式(16)可 解, 但多次求解问题式(16)计算成本是不能接受的。 因此利用代理模型是必要的, 即通过较少的采样获 取原问题信息, 构造一个计算成本较低的近似模型, 利用该模型求得近似的结果区间。结果的精度依赖 于所选的近似模型, 由于 Chebyshev 级数的优点, 本文选取该级数作为代理模型并由此得到 CSMM 方法。

本节将首先介绍如何求解一个函数的 Chebyshev 级数, 再将其作为代理模型分析原问题 的性态。

对于 $x \in \mathbf{R}$, 当 $x \in[-1,1]$ 时, $n$ 次 Chebyshev 多 项式 $C_{n}(x)$ 记为

$$
C_{n}(x)=\cos n \theta \quad \theta=\arccos (x)
$$

于是, $k$ 元函数 $\boldsymbol{f}(\boldsymbol{x})$ 的 $n$ 次截断 Chebyshev 级数可 表示为

$$
f(x) \approx p_{n}(x)=
$$

$\sum_{i_{1}=0}^{n} \sum_{i_{2}=0}^{n} \cdots \sum_{i_{k}=0}^{n}\left(\frac{1}{2}\right)^{p} f_{i_{1}, i_{2} \ldots, i_{k}} \cos \left(i_{1} \theta_{1}\right) \cos \left(i_{2} \theta_{2}\right) \cdots \cos \left(i_{k} \theta_{k}\right)$

式中, $p$ 为 $i_{l}=0, l=1,2, \cdots, k$ 的个数, $\theta_{i}=\arccos \left(x_{i}\right)$, $x_{i} \in[-1,1], i=1,2, \cdots, k$ 。若使用 Mehler 积分方法 ${ }^{[15-16]}$, $f_{i_{1}, i_{2}, \ldots, i_{k}}$ 可由下式确定

$$
\begin{gathered}
f_{i_{1}, i_{2} \ldots i_{k}}=\left(\frac{2}{\pi}\right)^{k} \int_{0}^{\pi} \int_{0}^{\pi} \cdots \int_{0}^{\pi} f\left(\cos \theta_{1} \cdots \cos \theta_{k}\right) \cos i_{1} \theta_{1} \cdots \\
\cos i_{k} \theta_{k} \mathrm{~d} \theta_{1} \cdots \mathrm{d} \theta_{k} \approx\left(\frac{2}{m}\right)^{k} \sum_{q_{1}=1}^{m} \cdots
\end{gathered}
$$




$$
\sum_{q_{k}=1}^{m} f\left(\cos \theta_{1}, \cdots, \cos \theta_{k}\right) \cos i_{1} \theta_{q_{1}} \cdots \cos i_{2} \theta_{q_{k}}
$$

式中 $m$ 为所取积分点个数, 为保证精度, 一般要求 $m \geqslant n+1$ 。其中

$$
\theta_{q_{i}}=\frac{2 q_{i}-1}{m} \frac{\pi}{2}, q_{i}=1,2, \cdots, m, i=1,2, \cdots, k
$$

上述运算是在每一自变量位于区间 $[-1,1]$ 内进 行的, 对于一般的区间, 若函数自变量表示为 $\xi \in \xi^{\mathrm{I}}=[\xi]=[\xi, \bar{\xi}]$, 对于 $k$ 元函数 $\boldsymbol{f}(\boldsymbol{\xi})$, 应计算 $\xi_{i}^{q}=\frac{\bar{\xi}_{i}+\underline{\xi_{i}}}{2}+\frac{\bar{\xi}_{i}-\underline{\xi_{i}}}{2} \cos \theta_{q}, i=1,2, \cdots, k$ 处的函数值, 再利用式(26)即可求得原函数对应的 Chebyshev 级 数, 代理模型亦完成构造。

为求得原函数的区间, 可以对 Chebyshev 代理 模型进行扫描, 得到近似的函数值区间。若对 Chebyshev 代理模型每一个自变量均选择 $e$ 个采样 点, 共需要 $e^{k}$ 个采样点, 用 $\tilde{\xi}_{i}^{j}, j=1,2, \cdots, e$ 表示第 $i$ 个自变量的第 $j$ 个采样点, 若 $\tilde{\xi}$ 表示采样点, 而 $\overline{\bar{\xi}}$ 表 示所有采样点构成的集合, 则有

$$
\begin{aligned}
& \min \boldsymbol{f}(\boldsymbol{\xi}) \approx \min \left\{\boldsymbol{p}_{n}(\tilde{\xi}) \mid \tilde{\xi} \in \overline{\bar{\xi}}\right\} \\
& \max \boldsymbol{f}(\boldsymbol{\xi}) \approx \max \left\{\boldsymbol{p}_{n}(\tilde{\xi}) \mid \tilde{\xi} \in \overline{\bar{\xi}}\right\}
\end{aligned}
$$

通过集合内的比较, 求得 $\min f(\xi)$, max $\boldsymbol{f}(\boldsymbol{\xi})$ 作为 原函数值区间的上下限, 则自变量区间 $[\xi]$ 对应的近 似函数值区间也就确定了。

由于上述过程对显式的 Chebyshev 级数进行扫 描, 即使采样点数 $e$ 较大, 也不会过多增加计算时 间。另一方面, 观察式(28), (27)可以发现, 求 Chebyshev 级数时, 上述过程仅需要 $m^{k}$ 个原函数值。 当原函数较复杂时, Chebyshev 代理模型就能大幅 降低计算时间。

对于式(22)的不确定性最优控制问题, 由于已 知 $\xi \in[\xi, \bar{\xi}]$, 可用 Chebyshev 代理模型求出性能 指标、输入控制力以及对应状态量的区间。不失 一般性, 可将整个优化过程看作求解一个关于可 变自变量 $\boldsymbol{\xi}$ 的矢量函数 $\boldsymbol{G}(\boldsymbol{\xi})$, 原目标函数最小值 $\min J$ 、输入控制 $\boldsymbol{u}$ 以及状态矢量 $\boldsymbol{x}$ 均可看作 $\boldsymbol{G}$ 的 分量

$$
\boldsymbol{G}(\xi)=\left(\begin{array}{c}
\min J \\
\boldsymbol{u}(\xi) \\
\boldsymbol{x}(\xi)
\end{array}\right)
$$

将 $\boldsymbol{G}$ 称为最优控制问题的响应。利用 Chebyshev 代 理模型, 可得对应 $\boldsymbol{G}(\boldsymbol{\xi})$ 的截断 Chebyshev 级数

$$
\boldsymbol{G}(\boldsymbol{\xi}) \Rightarrow \boldsymbol{G}_{C_{n}}(\boldsymbol{\xi})
$$

式中, $\boldsymbol{G}_{C_{n}}(\boldsymbol{\xi})$ 表示 $n$ 次截断 Chebyshev 级数, 与式 (26)类似，通过扫描 Chebyshev 代理模型，可近似 求出 $\boldsymbol{G}(\xi)$ 每一分量的最大值与最小值, 从而确定区 间矢量 $[\boldsymbol{G}]=[\underline{G}, \bar{G}]$, 最终得到 $[\min J]$, 及 $[\boldsymbol{u}],[\boldsymbol{x}]$, 至此含不确定参数的最优控制问题(22)完成求解。 此时求出了响应各分量的分布区间, 但一般而言, 各分量的区间不确定度是不同的。

\section{3 数值模拟}

为验证使用 Chebyshev 代理模型求解含不确定 参数吊车最优控制问题的可行性以及有效性, 本节 对具体的桥式吊车双摆模型进行分析与讨论。

与前述吊车模型相对应, 轨迹规划问题为: 给 定吊车启动与停止的时刻, 要求吊车运行过程中始 终满足事先规定的约束条件, 求出最节省能量的运 动方案, 并得到此时所需的输入控制力。系统参数 与约束条件如表 1 所示。

表 1 系统参数与约束条件

\begin{tabular}{lc}
\hline \multicolumn{1}{c}{ 系统参数 } & 名义值 \\
\hline 吊车质量 $M / \mathrm{kg}$ & 10 \\
吊钩质量 $m_{1} / \mathrm{kg}$ & 1 \\
重物质量 $m_{2} / \mathrm{kg}$ & 2 \\
质心距离 $1 l_{1} / \mathrm{m}$ & 0.7 \\
质心距离 $2 l_{2} / \mathrm{m}$ & 0.3 \\
重力加速度 $g /\left(\mathrm{m} / \mathrm{s}^{2}\right)$ & 9.8 \\
起始时刻 $t_{0} / \mathrm{s}$ & 0 \\
终止时刻 $t_{f} / \mathrm{s}$ & 6 \\
速度阈值 $v_{\max } /(\mathrm{m} / \mathrm{s})$ & 0.3 \\
控制力阈值 $u / \mathrm{N}$ & 80 \\
摆角 1 阈值 $\theta_{1 \max } /\left(^{\circ}\right)$ & 2 \\
摆角 2 阈值 $\theta_{2 \max } /\left(^{\circ}\right)$ & 2 \\
角速度 1 阈值 $\omega_{1 \max } /\left(\left(^{\circ}\right) / \mathrm{s}\right)$ & 5 \\
角速度 2 阈值 $\omega_{2 \max } /\left(\left(\left(^{\circ}\right) / \mathrm{s}\right)\right.$ & 5 \\
终止位置 $x_{f} / \mathrm{m}$ & 1 \\
\hline
\end{tabular}

状态量为 $\boldsymbol{x}=\left(x, \dot{x}, \theta_{1}, \dot{\theta}_{1}, \theta_{2}, \dot{\theta}_{2}\right)^{\mathrm{T}}$ ，选用能表征系 统能量消耗的性能指标 $J=\int_{t_{0}}^{t_{f}} u^{2} \mathrm{~d} t$ 。

实际吊车运行时, 吊车质量 $M$, 吊钩质量 $m_{1}$ 是 固定不变的, 而两者质心间的距离 $l_{1}$ 也是已知的, 但所吊重物的质量 $m_{2}$ 以及重物质心与吊钩质心的 距离 $l_{2}$ 往往不是固定值, 因此本文主要对 $m_{2}, l_{2}$ 含不 确定性的情况进行讨论与分析。 


\section{1 不确定性影响}

对重物质量存在 $5 \%\left(m_{2} \in[1.9,2.1] \mathrm{kg}\right)$ 、 $10 \%\left(m_{2} \in[1.8,2.2] \mathrm{kg}\right) 、 15 \%\left(m_{2} \in[1.7,2.3] \mathrm{kg}\right)$ 的不 确定度时, 吊车系统的最优控制方案进行分析。使 用 Chebyshev 代理模型法求解该不确定参数优化问 题时, Chebyshev 代理模型法的次数 $n=5$, 采样点 数 $m=8$ 。当重物存在 $5 \%$ 不确定度时, 各状态量 $x, v, \theta_{1}, \dot{\theta}_{1}, \theta_{2}, \dot{\theta}_{2}$ 以及输入控制力 $u$ 的结果区间如图 2 所示。

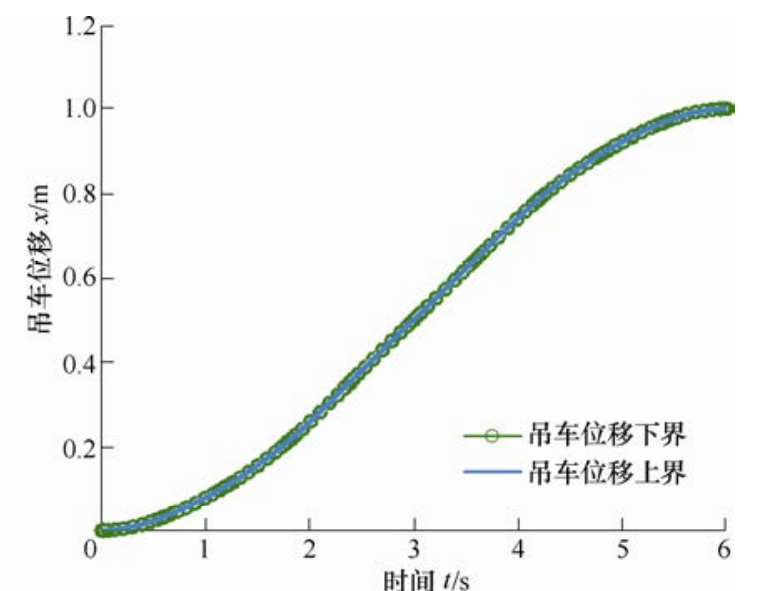

(a) 吊车位移区间

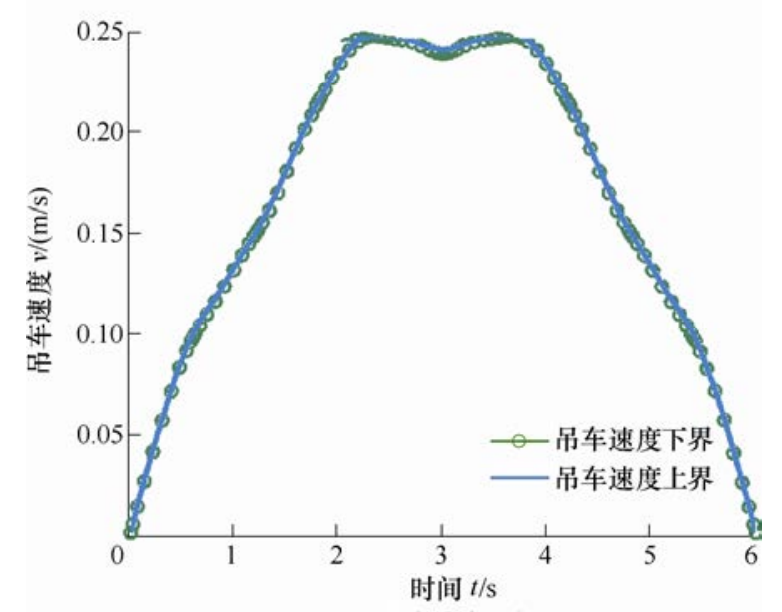

(b) 吊车速度区间

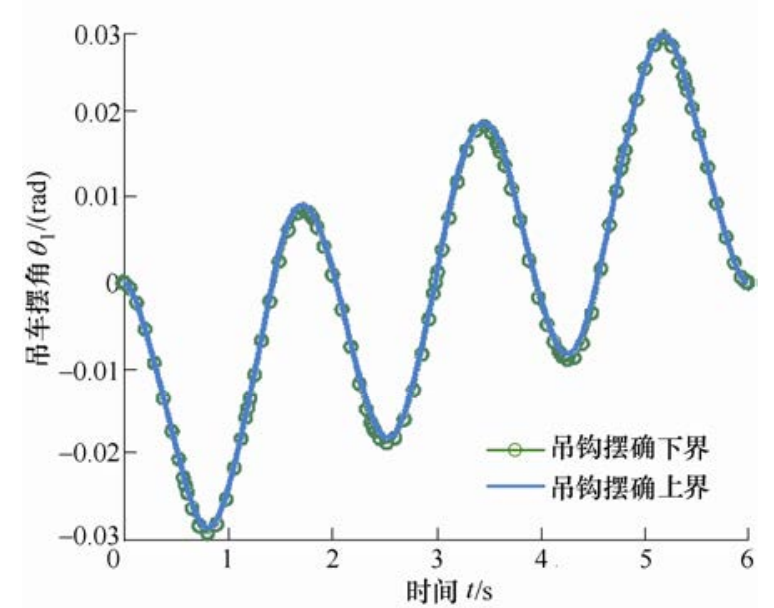

(c) 吊车吊钩摆角区间

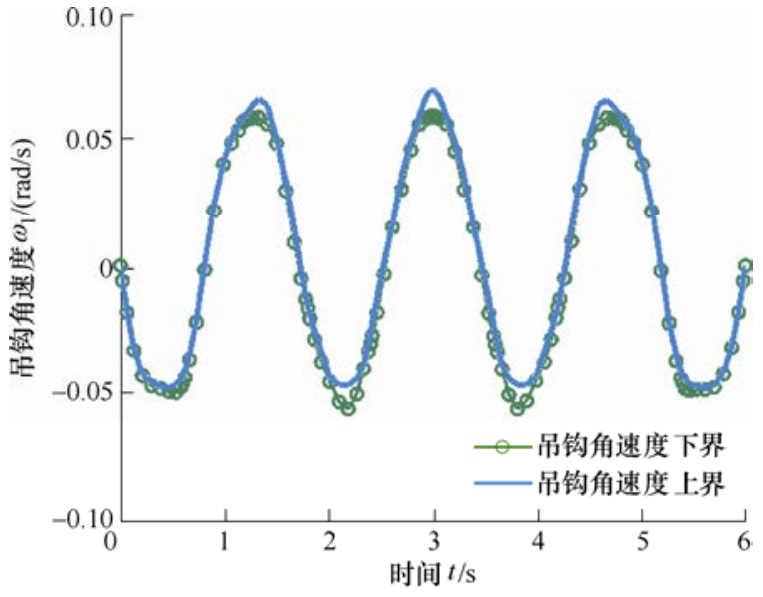

(d) 吊车吊钩角速度区间

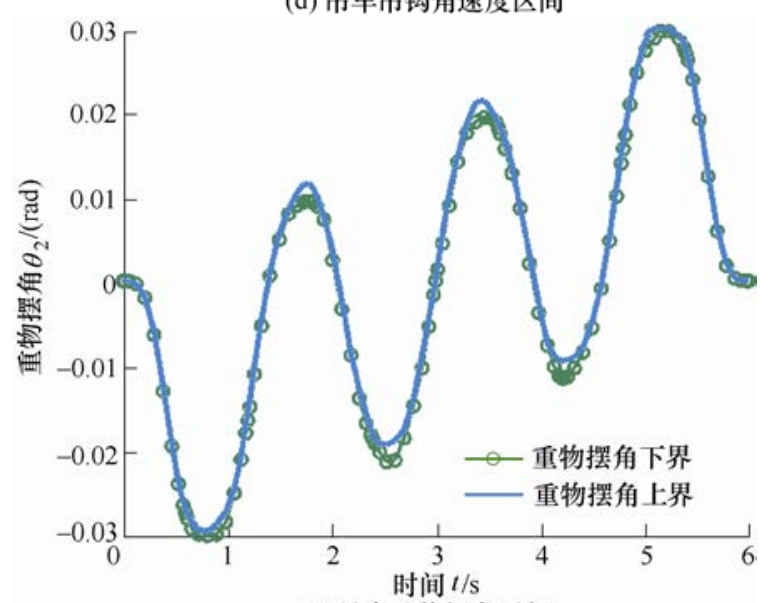

(e) 吊车重物摆角区间

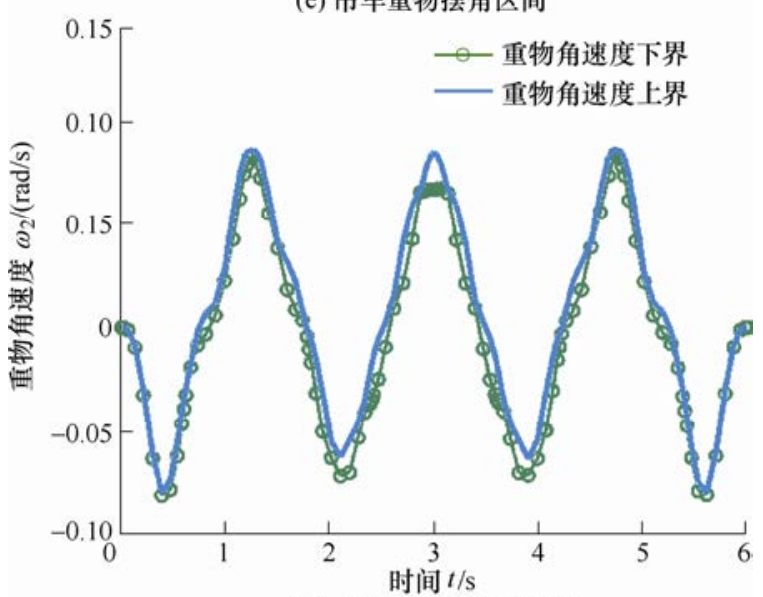

(f) 吊车重物角速度区间

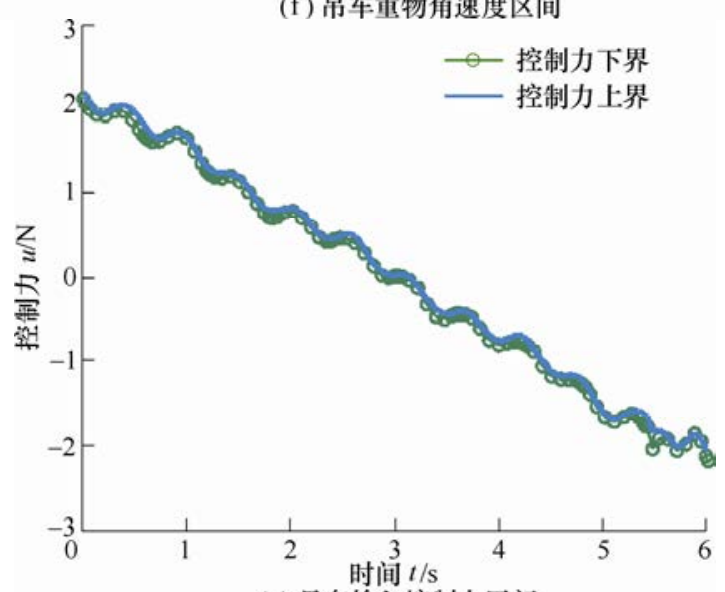

(g) 吊车输入控制力区间

图 2 吊车系统响应区间 
从图 2 中可以看到, 随着质量不确定性的引入, 状态矢量与控制力均存在不确定性, 但各量的不确 定程度又不尽相同。吊车的位移与速度几乎不受重 物质量的影响, 显得非常 “稳定”, 吊钩摆角、重物 摆角以及输入控制力则有一个狭窄的分布区间，而 吊钩角速度及重物角速度由于不确定性会产生明显 的变化, 显得非常 “敏感”。以重物角速度为例, 在重物质量不确定度变化时, 观察其变化。当重物 存在 $10 \%$ 不确定度时, 重物角速度如图 3 所示, 当 重物存在 15\%不确定度时, 重物角速度如图 4 所示。

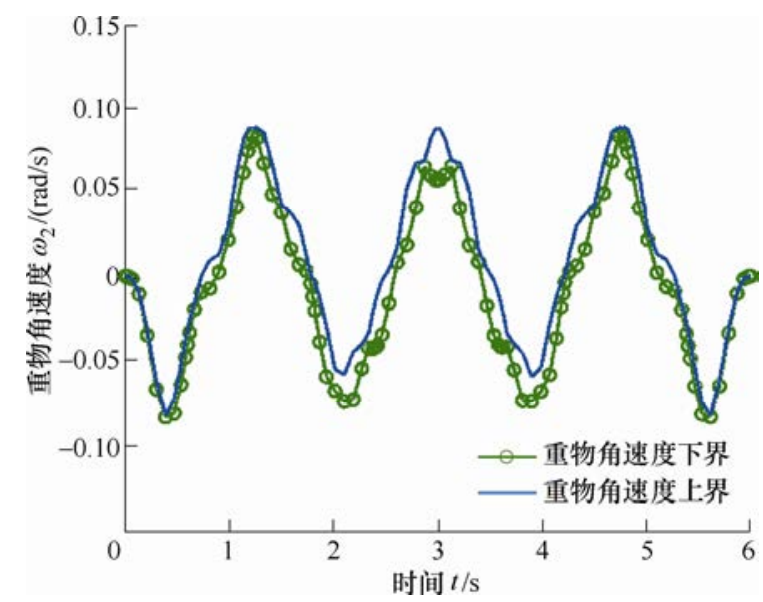

图 3 重物含 $10 \%$ 不确定度时重物角速度区间

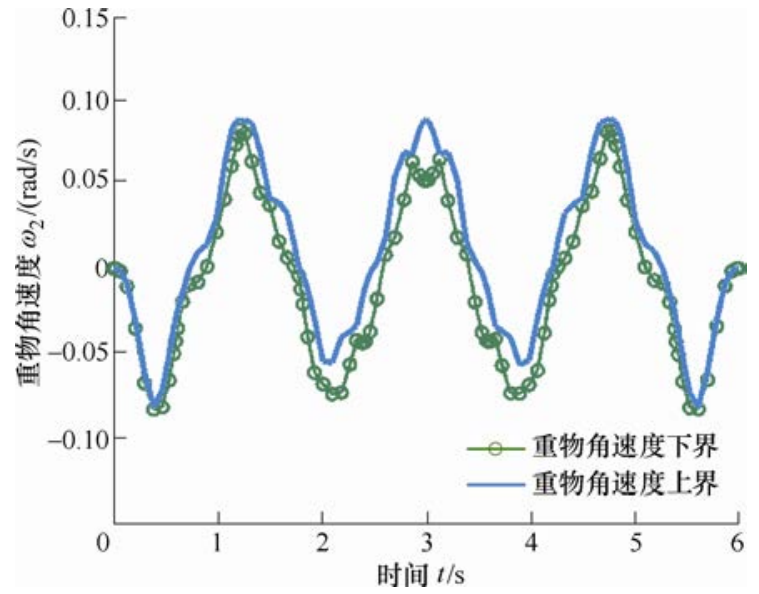

图 4 重物含 $15 \%$ 不确定度时重物角速度区间

为定量讨论不确定度大小对重物角速度的影 响, 选取 $t_{1}=1 \mathrm{~s}, t_{2}=3 \mathrm{~s}, t_{3}=5 \mathrm{~s}$ 三个时刻观察重物 角速度的不确定度, 结果如表 2 所示。由表 2 及 图 3、4 可知, 当重物不确定度一定时, 中间时刻 角速度的变化范围较大, 分布区间较宽, 而两侧 接近起始和终止位置, 区间逐渐变窄, 最后收缩 为一点。随着重物不确定度的增加, 重物角速度 的区间整体有变大的趋势，同时中间时刻的角速 度区间变化最为明显, 而两侧接近起始和终止位 置, 区间变化稍小。

表 2 特定时间点的重物角速度区间

\begin{tabular}{|c|c|c|c|c|c|c|}
\hline \multirow{2}{*}{ 时间点 } & \multicolumn{2}{|c|}{ 5\%不确定度 } & \multicolumn{2}{|c|}{ 10\%不确定度 } & \multicolumn{2}{|c|}{ 15\%不确定度 } \\
\hline & 区间 & 不确定度(\%) & 区间 & 不确定度(\%) & 区间 & 不确定度(\%) \\
\hline$t_{1}$ & {$[0.0228,0.0282]$} & 10.5 & {$[0.0216,0.0292]$} & 15.0 & {$[0.0216,0.0292]$} & 15.0 \\
\hline$t_{2}$ & {$[0.0678,0.0857]$} & 11.7 & {$[0.0564,0.0873]$} & 21.5 & {$[0.0518,0.0875]$} & 25.6 \\
\hline$t_{3}$ & {$[0.0225,0.0270]$} & 9.1 & {$[0.0214,0.0274]$} & 12.3 & {$[0.0214,0.0274]$} & 12.3 \\
\hline
\end{tabular}

从上述分析可以看出, Chebyshev 代理模型法 可以求解含有不确定度的吊车最优控制问题, 同时 也能看出, 不确定度对各状态量影响程度是不同的。

\section{2 误差分析}

前文证明 Chebyshev 代理模型求解不确定问题 是可行的, 本小节则将对其求解精度进行分析。此 处使用扫描遍历法、Chebyshev 代理模型法和 Chebyshev 扩张函数法 ${ }^{[15-16]}$ 分别求解不确定性吊车 最优控制问题, 对比三种方法的误差。为方便比较, 可认为使用扫描遍历法所得到的区间为真实区间, 而将其他方法的结果与之比较。

求解时, 重物质量存在 $10 \%$ 不确定度, 即 $m_{2} \in[1.80,2.20] \mathrm{kg}$, 其余吊车参数同表 1 , 扫描遍 历法的采样点数选为 30, Chebyshev 代理模型法与 Chebyshev 扩张函数法的次数 $n=5$, 采样点数为 $m=8$ 。对比时以重物角速度区间为例, 图 5 给出 了扫描遍历法与 Chebyshev 代理模型法的区间对 比。图 6 则给出了扫描遍历法与 Chebyshev 扩张函 数法的区间对比。

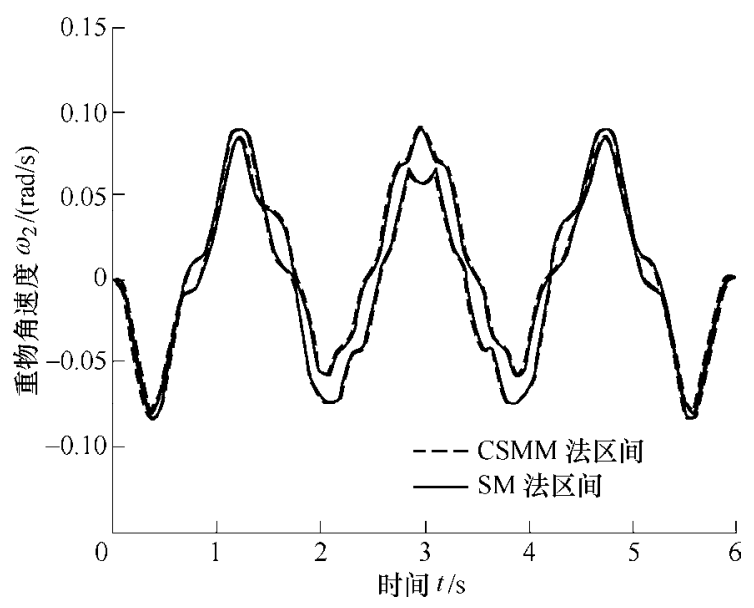

图 5 重物角速度区间对比 1 


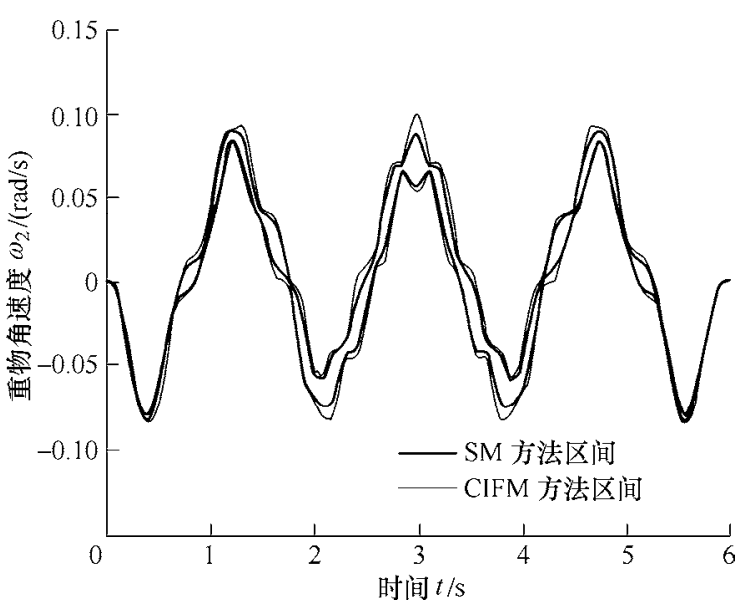

图 6 重物角速度区间对比 2

相比较而言, 代理模型法所得区间结果更接近 扫描遍历法的结果, 比扩张函数法更为精确。为仔 细比较三种方法所得结果, 更精确地看出区间差异, 定义如下区间误差值

$$
\Delta=\left(x_{s}^{u}-x_{s}^{l}\right)-\left(x_{c}^{u}-x_{c}^{l}\right)
$$

式中, $x_{s}^{u}$ 代表使用扫描遍历法得到的变量上限, $x_{s}^{l}$ 代表使用扫描遍历法得到的变量下限, $x_{c}^{u}$ 代表使用 Chebyshev 代理模型法或 Chebyshev 扩张函数法得 到的变量上限, $x_{c}^{l}$ 代表使用 Chebyshev 代理模型法 或 Chebyshev 扩张函数法得到的变量下限。对重物 角速度, 在重物存在 5\%、10\%、15\%不确定度时分 别用 Chebyshev 代理模型法和 Chebyshev 扩张函数 法计算重物角速度分布区间, 误差结果如图 7、8 所示。

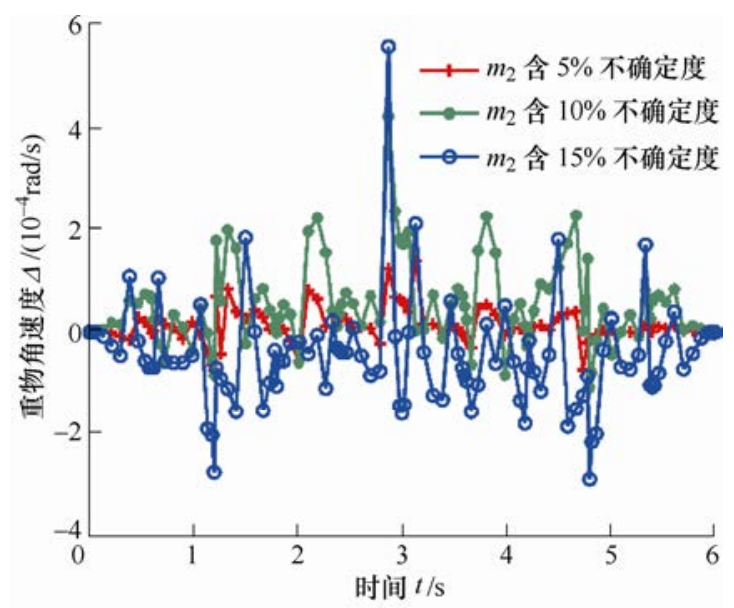

图 7 Chebyshev 代理模型法所得角速度误差

从图 8 可以发现, 随着不确定度的增大, 使用 Chebyshev 扩张函数求解时, 角速度误差也会不断 增大，当重物质量不确定度达 15\%时，角速度区间 最大误差的绝对值已达 0.024。同时图 8 中 $\Delta$ 值均为 负数, 说明该方法所得区间一直比真实区间偏大。 反之观察图 7, 随着不确定度的增大，除中间时刻

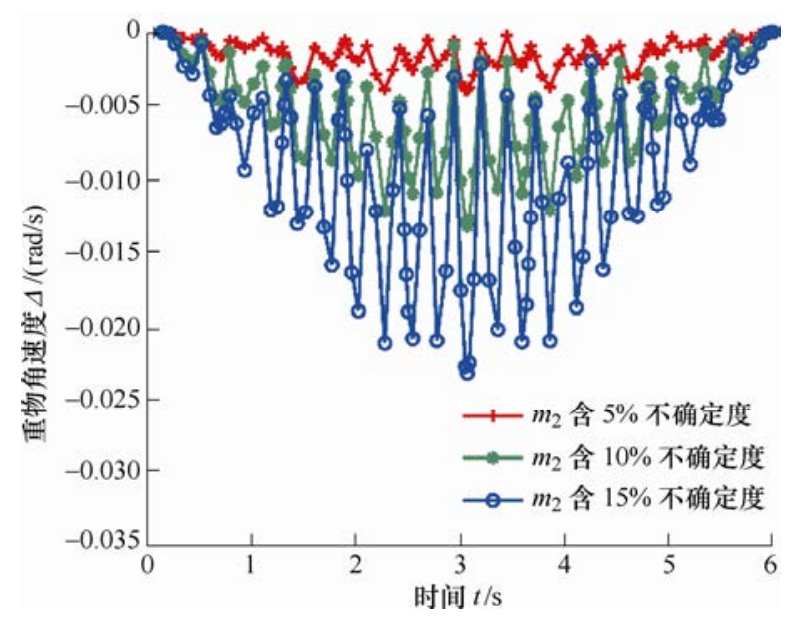

图 8 Chebyshev 扩张函数法所得角速度误差

误差迅速增大外，误差增大较为缓慢。同时最大区 间误差不超过 0.000 6, 不到 Chebyshev 扩张函数法 的 $1 / 40$, 而 $\Delta$ 值有正有负，说明该方法所得区间更 接近真实区间。总体来说, 两种方法均在吊车运行 的中间时刻存在最大误差，但 Chebyshev 代理模型 法的误差整体较小，且不会由于不确定度的变化而 剧烈变大, 所得结果更令人满意。

\section{3 计算效率}

通过前两节的分析可以看出, 使用 Chebyshev 代理模型法求解含不确定参数的吊车最优控制问题 是可行的, 同时对比可发现其计算精度较高, 所得 结果区间相较之 Chebyshev 扩张函数法更接近扫描 遍历法的结果。扫描遍历法能求到最好的结果, 但 计算成本非常高。相比之下 Chebyshev 代理模型法 和 Chebyshev 扩张函数法所需采样点不多, 总体计 算时间可以大大缩短。以下对三种方法的计算时间 进行对比。选取两个模型进行讨论。

模型 1 : 仅有重物质量 $m_{2}$ 存在不确定度, 吊车 系统各参数取表 1 的结果，重物质量存在 $15 \%$ 的不 确定度, $m_{2} \in[1.7,2.3] \mathrm{kg}$ 。计算时, 采样点遍历法 使用 30 个采样点, Chebyshev 代理模型法和 Chebyshev 扩张函数的采样点均选为 8 个, 级数次 数均选为 5 。

模型 2: 重物质量 $m_{2}$ 以及重物质心与吊钩间的 距离 $l_{2}$ 均存在不确定度, 即存在两个不确定变量, 吊车各参数仍选取表 1 的结果，重物质量存在 $15 \%$ 的不确定度， $m_{2} \in[1.7,2.3] \mathrm{kg}$, 而 $l_{2}$ 存在 $5 \%$ 不确 定度, $l_{2} \in[0.29,0.31] \mathrm{m}$ 。计算时, 扫描遍历法对每 一自变量选取 30 个采样点, 共选取 900 个采样点。 而 Chebyshev 代理模型法与 Chebyshev 扩张函数法 对每一不确定变量选取 8 个采样点, 一共选取 64 个采样点, 级数次数均选为 5 。

计算时使用 Intel i7-4710HQ(2.5 GHz)处理器, 
内存为 $8 \mathrm{~GB}$, 操作系统为 Windows 7(64 位), Matlab 版本为 R2010a。三种方法的计算时间如表 3 所示, 由表中可以看出, 当仅有一个不确定变量时, Chebyshev 代理模型法计算时间仅为扫描遍历法计 算时间的 0.27 倍, 对比 Chebyshev 扩张函数法, 其 计算时间并没有明显变长。当问题中含有两个不确 定变量时, 采样点遍历法计算时间过长, 而 Chebyshev 代理模型法计算所用时间仅为扫描遍历 法所用时间的 0.07 倍, 同时仅仅比 Chebyshev 扩张 函数法慢 $1.56 \mathrm{~s}$ 。

\begin{tabular}{|c|c|c|c|}
\hline 模型 & 采样点遍历法 & $\begin{array}{l}\text { Chebyshev } \\
\text { 扩张函数法 }\end{array}$ & $\begin{array}{l}\text { Chebyshev } \\
\text { 代理模型法 }\end{array}$ \\
\hline 模型 1 & 146.49 & 39.08 & 39.45 \\
\hline 模型 2 & 4966.00 & 345.47 & 347.03 \\
\hline
\end{tabular}

综合上述分析, 扫描遍历法虽能求出最好的结 果, 但随着不确定变量数的增加, 计算时间过长; Chebyshev 扩张函数法能极大缩短计算时间, 但所 得结果区间往往偏大, 精度不够理想; 而本文所提 到的 Chebyshev 代理模型法, 计算时间仅比 Chebyshev 扩张函数法稍长, 但计算精度却远高于 前者, 更接近扫描遍历法的结果, 可以说是在精度 和计算时间上做了平衡与折衷。

\section{4 结论}

针对目前考虑不确定性吊车轨迹规划存在的困 难, 本文提出一种基于 Chebyshev 代理模型的吊车 不确定性轨迹规划求解方法。

(1) 使用非概率区间模型描述不确定性, 在考 虑不确定性时, 吊车运动轨迹以区间表示。整个方 法求解时无需系统的概率信息, 所得吊车轨迹区间 包含所有可能的轨迹, 清唽直观地反映了不确定性 对吊车系统的影响。

(2) 利用保辛伪谱方法求解确定性最优控制问 题并作为样本, 使用截断 Chebyshev 级数作为代理 模型近似代替原非线性最优控制问题, 仅需较少的 采样数据, 代理模型就能足够接近原最优控制问题。 利用扫描代理模型的方式得到响应量的不确定性分 布区间。由于对显式的代理模型进行扫描, 因此计 算时间不会大幅增加。

(3) 相较于采样点遍历法, 所提出的 CSMM 方 法仅需较少的采样点, 因而计算成本大幅降低, 而 相较于 Chebyshev 扩张函数法, 数值算例表明本文 方法计算精度更高, 所得区间结果一定程度上揭示
了双摆吊车模型参数与状态量的内在关系。对于实 际吊车系统, 可利用本文方法估计不确定性参数对 吊车系统的影响, 为吊车系统鲁棒性与稳定性研究 提供参考。

\section{参 考 文 献}

[1] VAUGHAN J, KIM D, SINGHOSE W. Control of tower cranes with double-pendulum payload dynamics[J]. IEEE Transactions on Control Systems Technology, 2010, 18(6): 1345-1358.

[2] LIU Yang, YU Hongnian. A survey of underactuated mechanical systems[J]. IET Control Theory \& Application, 2013, 7(7): 921-935.

[3] FANG Yongchun, MA Bojun, WANG Pengcheng, et al. A motion planning-based adaptive control method for an underactuated crane system[J]. IEEE Transactions on Control Systems Technology, 2012, 20(1): 241-248.

[4] ABDEL-RARMAN E, NAYFEH A H, MASOUD Z N. Dynamics and control of cranes: a review[J]. Journal of Vibration and Control, 2003, 9(7): 863-908.

[5] 史晓佳, 张福民, 曲兴华, 等. KUKA 工业机器人位姿 测量与在线误差补偿[J]. 机械工程学报, 2017, 53(8): 1-7.

SHI Xiaojia, ZHANG Fumin, QU Xinghua, et al. Position and attitude measurement and online errors compensation for KUKA industrial robots[J]. Journal of Mechanical Engineering, 2017, 53(8): 1-7.

[6] ZHANG Xuebo, FANG Yongchun, SUN Ning. Minimum-time trajectory planning for underactuated overhead crane systems with state and control constraints[J]. IEEE Transactions on Industrial Electronic, 2014, 61(12): 6915-6925.

[7] WU Zhou. XIA Xiaohua. Optimal motion planning for overhead cranes[J]. IET Control Theory \& Application, 2014, 8(17): 1833-1842.

[8] 孙宁, 方勇纯, 钱或哲. 带有状态约束的双摆效应吊车 轨迹规划 $[\mathrm{J}]$. 控制理论与应用, 2014, 31(7): 974-980. SUN Ning, FANG Yongchun, QIAN Yuzhe. Motion planning for cranes with double pendulum effects subject to state constraints[J]. Control Theory \& Applications, 2014, 31(7): 974-980.

[9] 陈鹤，方勇纯，孙宁，等. 基于伪谱法的双摆吊车时间 最优消摆轨迹规划策略 $[\mathrm{J}]$. 自动化学报, 2016, 42(1): 153-160.

CHEN He, FANG Yongchun, SUN Ning, et al. Pseudospectral method based time optimal anti-swing trajectory planning for double pendulum crane systems[J]. Acta Automatica Sinica, 2016, 42(1): 153-160. 
[10] WU Zhou, XIA Xiaohua. Energy efficiency of overhead cranes[C]/Proceedings of the 19th World Congress-the International Federation of Automatic Control. Cape Town, South Afican: Elsevier Science BV, 2014: 19-24.

[11] 刘殿通, 易建强, 谭民. 不确定欠驱动非线性系统的模 糊滑模控制[J]. 电机与控制学报, 2003, 7(3): 215-218. LIU Diantong, YI Jianqiang, TAN Min. Fuzzy sliding mode control of uncertain underactuated nonlinear systems[J]. Electric Mechines and Control，2003， 7(3): 215-218.

[12] TUAN L A, LEE S G, KO D H, et al. Combined control with sliding mode and partial feedback linearization for 3D overhead craned[J]. International Journal of Robust And Nonlinear Control, 2014(24): 3372-3386.

[13] ALEFELD G, MAYER G. Interval analysis: theory and applications[J]. Journal of Computational and Applied Mathematics, 2000, 121: 421-464.

[14] LIU Zhuangzhuang, WANG Shutian, LI Junfei. Nonintrusive hybrid interval method for uncertain nonlinear systems using derivative information[J]. Acta Mechanica Sinica, 2015, 32(1): 170-180.

[15] WU Jinglai, ZHANG Yunqing, CHEN Liping, et al. A Chebyshev interval method for nonlinear dynamics systems under uncertainty[J]. Applied Mathematical Modelling, 2013, 37(6): 4578-4591.

[16] WU Jinglai, LUO Zhen, ZHANG Yunqing, et al. Interval uncertain method for multi-body mechanical systems based on Chebyshev inclusion functions[J]. International Journal for Numerical Methods in Engineering, 2013, 95(7): 608-630.

[17] LI Yunlong, WANG Xiaojun, HUANG Ren, et al. Actuator placement robust optimization for vibration control system with interval parameters[J]. Aerospace Science and Technology, 2015， 45: 88-98.

[18] PENG Haijun, WANG Xinwei, LI Mingwu, et al. An hp symplectic pseudospectral method for nonlinear optimal control[J]. Communications in Nonlinear Science and Numerical Simulation, 2017, 42: 623-644.

作者简介: 彭海军(通信作者), 男, 1982 年出生, 博士, 副教授。主要 研究方向为载运工具动力学与控制、计算最优控制数值方法。

E-mail: hjpeng@dlut.edu.cn

施博洋, 男, 1994 年出生。主要研究方向为机器人不确定性路径规划与 运动控制。

E-mail: sby777@mail.dlut.edu.cn

王听炜, 男, 1992 年出生, 博士研究生。主要研究方向为非线性最优控 制的保辛方法。

E-mail: wangxinwei@mail.dlut.edu.cn 